\title{
The Significance of an Unnoticed Theoretical Element
}

\author{
Eliahu Comay \\ Charactell Ltd., Tel Aviv, Israel \\ Email: elicomay@post.tau.ac.il
}

How to cite this paper: Comay, E. (2020) The Significance of an Unnoticed Theoretical Element. Open Access Library Journal, 7: e6262.

https://doi.org/10.4236/oalib.1106262

Received: March 24, 2020

Accepted: April 12, 2020

Published: April 15, 2020

Copyright $\odot 2020$ by author(s) and Open Access Library Inc.

This work is licensed under the Creative Commons Attribution International License (CC BY 4.0).

http://creativecommons.org/licenses/by/4.0/

\begin{abstract}
Relativistic properties of a Lagrangian density are compared with those of a Hamiltonian density. It is proved that a Lagrangian density and a Hamiltonian density undergo different Lorentz transformations. This outcome is a theoretical element that has been unnoticed for a very long time. It is also proved that this theoretical element plays a crucial role in the structure of weak interactions theory. In particular, it is shown that the theory that uses this element is overwhelmingly superior over the Standard Model electroweak theory.
\end{abstract}

\section{Subject Areas}

Mechanics, Modern Physics

\section{Keywords}

Lagrangian Density, Hamiltonian Density, Lorentz Transformations, Weak Interactions

\section{Introduction}

Physics is a science that aims to describe the state and the time-evolution of natural systems that belong to its domain of validity. On the other hand, during the last four centuries, physical laws take a mathematical form [1]. It means that any physical theory must have a coherent mathematical structure. At present, there are quite a few laws that any given physical theory should abide with. For example, there are conservation laws like those of energy, momentum, angular momentum, electric charge, baryonic number and leptonic number that impose constraints on any given physical theory. It means that if the final result of a new physical theory violates any of these conservation laws then this theory should be rejected. 
The progress of mathematical physics has yielded principles where each of which may be used as a solid element of a new physical theory. For example, the variational principle is now regarded as a solid basis that a given quantum theory may use. Indeed, it is stated that "all field theories used in current theories of elementary particles have Lagrangians of this form" (see [2], p. 300). Here the action takes the form

$$
I(\psi)=\int \mathrm{d}^{4} x \mathcal{L}(\psi, \psi, \mu),
$$

where $\psi$ is the quantum function, the action $I(\psi)$ is a Lorentz scalar, and $\mathcal{L}\left(\psi, \psi_{, \mu}\right)$ is the Lagrangian density of a given theory.

The Hamiltonian is another important quantity that quantum theories use. The Hamiltonian density of a given quantum theory is obtained from the Legendre transformation of its Lagrangian density (see [2], p. 301)

$$
\mathcal{H}_{D}=\pi \dot{\psi}-\mathcal{L} .
$$

Here $\pi$ denotes the generalized momentum and the upper dot denotes the time-derivative.

This work examines relationships between the Lagrangian density of a quantum theory and its Hamiltonian density. A quite straightforward proof shows that terms of a Lagrangian density and terms of its Hamiltonian density undergo different relativistic transformations. This property is called below the Lagrangian-Hamiltonian Covariance Difference (LHCD). It turns out that the LHCD, which is unnoticed by current textbooks, is relevant to the theoretical structure of weak interactions.

Units where $\hbar=c=1$ are used. Greek indices run from 0 to 3 . Most formulas take the standard form of relativistic covariant expressions. The metric is diagonal and its entries are $(1,-1,-1,-1)$. An upper dot denotes the time-derivative. Section 2 discusses the LHCD. Section 3 shows that the LHCD plays a crucial role in a construction of a consistent weak interaction theory. Section 4 proves the overwhelming advantage of the weak interaction theory that is based on the LHCD over the Standard Model's electroweak theory. The last section summarizes this work.

\section{An Unnoticed Theoretical Element}

A comparison between a Lagrangian and its associated Hamiltonian illuminates a theoretical element that is unnoticed in the literature. Consider for example the Lagrangian of a classical charged particle interacting with electromagnetic fields (see [3], pp. 48, 49)

$$
L=-m \sqrt{1-v^{2}}+e \mathbf{A} \cdot \boldsymbol{v}-e \phi,
$$

where $A^{\mu}=(\phi, \boldsymbol{A})$ is the 4 -potential of the electromagnetic fields. The Hamiltonian that is derived from (3) is

$$
H=\boldsymbol{v} \cdot \frac{\partial L}{\partial \boldsymbol{v}}-L=\frac{m}{\sqrt{1-v^{2}}}+e \phi .
$$


This Hamiltonian should be rewritten as a function of the coordinates and the generalized momentum. Here the generalized momentum is

$$
\boldsymbol{P}=\frac{m \boldsymbol{v}}{\sqrt{1-v^{2}}}+e \boldsymbol{A}
$$

One may inspect the interaction term of (3) and (4) and conclude that in the classical case velocity independent terms of the Lagrangian are the same as those of the Hamiltonian, but with opposite sign.

The main issue of this work pertains to the quantum domain. Quantum theories are derived from a Lagrangian density. It turns out that the literature overlooks this statement: Contrary to an impression based on the previous conclusion, covariant properties of an interaction term of a Lagrangian density are not the same as those of the Hamiltonian density.

Applying the action (1), one derives the proof in a quite straightforward manner. A Lorentz invariant theory of a quantum field $\psi$ is based on a Lorentz scalar action, where $\mathcal{L}\left(\psi, \psi_{, \mu}\right)$ is the theory's Lagrangian density. Here the product $\mathrm{d}^{4} x$ of the differentials of (1) is a Lorentz scalar. Hence, also the Lagrangian density $\mathcal{L}(\psi, \psi, \mu)$ is a Lorentz scalar. On the other hand, the Hamiltonian is an energy operator, which transforms like the 0 -component of a 4-vector, and density is the 0 -component of the 4-current. Hence, the Hamiltonian density is the 00-component of a second rank tensor.

Conclusion: the Lagrangian density and the Hamiltonian density have different covariant properties.

As stated above, this conclusion is called herein the Lagrangian-Hamiltonian Covariance Difference (LHCD).

Here is an example that illustrates the LHCD validity. Consider the Dirac Lagrangian density

$$
\mathcal{L}_{D}=\bar{\psi}\left(\gamma^{\mu} i \partial_{\mu}-m\right) \psi-e \bar{\psi} \gamma^{\mu} A_{\mu} \psi
$$

(see [4], p. 84, [5], p. 78). The last term represents the electromagnetic interaction of a charged Dirac particle.

The Legendre transformation of (6) yields the Dirac Hamiltonian density

$$
\mathcal{H}_{D}=\pi \dot{\psi}-\mathcal{L}=\psi^{\dagger}[\boldsymbol{\alpha} \cdot(-i \nabla-e \boldsymbol{A})+\beta m+e \phi] \psi,
$$

where $\alpha, \beta$ are the ordinary Dirac matrices, and the relation $\bar{\psi}=\psi^{\dagger} \gamma^{0}$ is used (see [4], p. 87). The product $\psi^{\dagger} \psi$ of (7) is the Dirac density, and the quantities inside the square brackets compose the Dirac Hamiltonian operator. The Dirac equation is (see [6], p. 11)

$$
i \frac{\partial \psi}{\partial t}=H \psi=[\boldsymbol{\alpha} \cdot(-i \nabla-e \boldsymbol{A})+\beta m+e \phi] \psi,
$$

Evidently, $\phi$ is the 0 -component of the 4 -potential, and in the Dirac Lagrangian density (6) it is multiplied by the matrix $\gamma^{0}$. By contrast, no matrix multiplies the corresponding term of the Dirac Hamiltonian density (7). The matrix $\gamma^{0}$ is the 0 -component of the 4 -vector $\gamma^{\mu}$. It means that interaction 
terms of (6) and (7) take a different covariant structure. This result is in accordance with the LHCD.

The following argument illuminates another facet of this outcome. The product $\bar{\psi} \psi$ is a Lorentz scalar (see [6], p. 26). Therefore, also the terms of the Lagrangian density (6) that are enclosed between $\bar{\psi}, \psi$ should be Lorentz scalars. On the other hand, $\psi^{\dagger} \psi \quad$ (where $\psi^{\dagger}=\bar{\psi} \gamma^{0}$ ) is the density of the Dirac particle, which is the 0-component of the Dirac 4-current (see [6], pp. 23-24). Furthermore, the Hamiltonian must be written in terms of the generalized momentum, and the generalized momentum associated with the Dirac function $\psi$ is (see [4], p. 55; [5], p. 52)

$$
\pi_{D}=\frac{\partial \mathcal{L}}{\partial \dot{\psi}}=i \bar{\psi} \gamma^{0}=i \psi^{\dagger} .
$$

It follows that the Dirac Hamiltonian density (7) is written in terms of $\psi$, which is regarded as the generalized coordinate, and $\psi^{\dagger}$, where (9) is the Dirac generalized momentum. This is the Hamiltonian's standard structure, which is a function of generalized coordinates and generalized momenta.

It is shown later in this work that the distinction between terms of the Lagrangian density and corresponding terms of the Hamiltonian is relevant to the theoretical analysis of weak interactions.

\section{An Alternative History of the Weak Interaction Theory}

Landmarks of the historical progress of the weak interaction theory are mentioned below.

1) Fermi proposed in 1934 a weak interaction theory aiming to describe the $\beta$ decay of nuclei (see e.g. [7], p. 296). Figure 1 illustrates the neutron's $\beta$ decay (see [8], p. 209). This figure shows that four fermions are involved in this weak process. The Fermi theory is based on a 4 -fermions contact interaction, where a constant $G_{F}$ whose dimension is $\left[L^{2}\right]$ describes the interaction strength. The Fermi $\beta$ decay allows no change of the spin of the initial nuclear state and that of the final nuclear state.

2) A few years later Gamow and Teller extended Fermi's work, and their theory allows a $\beta$ transition where the spin of the two nuclei differs by unity. Experiments show that the strength of the Gamow-Teller transition is somewhat stronger than that of the Fermi transition

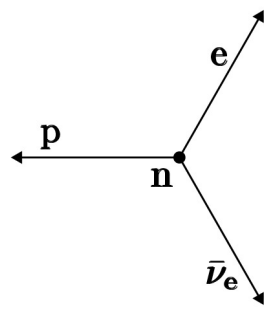

Figure 1. An Illustration of the neutron weak decay. Arrows denote the outgoing particles' momentum. 


$$
G_{A} \square 1.3 G_{V},
$$

where $G_{V}, G_{A}$ denote the strength of the Fermi and the Gamow-Teller transitions, respectively (see [9], p. 188).

3) About 20 years later Lee and Yang proposed the idea that weak interactions violate parity conservation. In 1957, namely, soon after the publication of this idea, $\mathrm{Wu}$ et al. carried out an experiment that proved parity violation in the $\beta$ decay of the ${ }^{60}$ Co nucleus (see [8], p. 214).

4) The Wu experiment has motivated Sudarshan and Marshak to examine the available weak decay data. They concluded that the weak interaction can be described as the difference between a vector and axial-vector interactions (called V-A) (see [8], pp. 217-220).

5) Feynman and Gel-Mann published in 1958 an article that presents a theoretical progress of the weak interaction problem [10]. Their work relies on the idea of a massless neutrino. They also introduced the $\left(1 \pm \gamma^{5}\right)$ factor to the equations of motion. Here the pure number 1 is a scalar and the $\gamma^{5}$ matrix is a pseudoscalar (see [6], p. 26). Hence, $\left(1 \pm \gamma^{5}\right)$ is a maximal parity violation operator. This operator complies with the weak interactions V-A attribute of Sudarshan and Marshak.

6) Glashow, Salam and Weinberg formulated the electroweak theory during the next decade. This is a field theory that combines electrodynamics and weak interactions. This theory uses the factor $\left(1 \pm \gamma^{5}\right)$, and the fields of the $W^{ \pm}, Z$ massive bosons mediate the weak interactions (see e.g. [7], Chapter 15).

Let us try to show an alternative course of the actual progress of the weak interaction theory. This course departs from the actual course at point 5 (Item 5' replaces Item 5, etc.) It relies on the experimental and theoretical elements that were known in 1958.

5’) The English translation of Wentzel's QFT textbook [11] was published in 1949. It explains how one can prove that conservation laws are satisfied by a QFT theory that is based on the variational principle. This approach is now regarded as the standard route for a QFT construction. Indeed, as stated in the introduction, "all field theories used in current theories of elementary particles have Lagrangians of this form" (see [2], p. 300). The LHCD element, which is described in the previous section, is a straightforward outcome of this QFT structure. Hence, it is assumed that the LHCD has been discovered before 1958.

6 ') The Fermi golden rule applies to the $\beta$ decay. This rule uses the Hamiltonian of the system for a description of the transition between an initial state and a final state. Hence, the structure of the Hamiltonian is required for a theoretical description of a weak decay. This point emphasizes the relevance of the LHCD to the analysis of weak interaction data.

$\left.7^{\prime}\right)$ Figure 1 shows that an electron and a neutrino are produced in a $\beta$ decay. At that time the electron was known much better than the neutrino. Hence, one naturally favors a weak interaction theory that relies on an appropriate extension of the electron's Lagrangian density (6). It means that the main problem is: 
What is the form of the weak interaction term that should be added to the electron's Lagrangian density, whose Hamiltonian abides by the experimentally known properties of weak interactions? These properties are mentioned below:

7'a) The interaction strength should have the dimension $\left[L^{2}\right]$ of the Fermi constant $G_{F}$.

7’b) The associated Hamiltonian should comply with the V-A property of weak interactions.

8') In 1941 Pauli examined the following interaction term of the electron

$$
\mathcal{L}^{\prime}=d \bar{\psi} \sigma_{\mu \nu} F^{\mu v} \psi
$$

where $F^{\mu v}$ is the electromagnetic field tensor, and the coefficient $d$ has the dimension of length. This term is called tensor interaction, due to its dependence on $\sigma_{\mu \nu}$. The interaction (11) alters the Dirac expression for the magnetic dipole moment of the electron (see [2], p. 14; [12], p. 223). However, the ordinary Dirac Lagrangian density (6), which contains no term like (11), yields a very good prediction for the electron's magnetic dipole moment. Hence, the Pauli term (11) has been abandoned as a term that pertains to the electron's electromagnetic interaction.

It can be proved that the Pauli term (11) is the required weak interaction term of the Lagrangian density (see [13] [14] [15]). Here the external field $F^{\mu v}$ is a Maxwellian-like weak field of a weak dipole that is associated with the spin of an external Dirac particle. Therefore, the coefficient that describes the interaction strength has the dimension $\left[L^{2}\right]$. Moreover, a proof showing that the associated Hamiltonian abides by the $\mathrm{V}$-A property of weak interactions is shown in [13] [14] [15]). The Lagrangian density of the alternative theory is

$$
\mathcal{L}_{D}=\bar{\psi}\left(\gamma^{\mu} i \partial_{\mu}-m\right) \psi-e \bar{\psi} \gamma^{\mu} A_{\mu} \psi+d \bar{\psi} \sigma_{\mu v} \mathcal{F}^{\mu v} \psi,
$$

where $\mathcal{F}^{\mu v}$ is the tensor of the Maxwellian-like weak field that is associated with a weak dipole. The Lagrangian (12) is an extension of the Dirac Lagrangian density (6), and the last term of (12) represents weak interactions. This theory is called below Dipole-Dipole Weak Interaction Theory(DDWIT).

$\left.9^{\prime}\right)$ The actual historical course of the weak interaction uses the $\left(1 \pm \gamma^{5}\right)$ factor for imposing the $\mathrm{V}$-A attribute. By contrast, the alternative route uses general principles of physics where the Hamiltonian that is derived from the Lagrangian density (12) proves the V-A weak interactions attribute.

10 ') Conclusion: The alternative historical progress which is described in Items 5' - 9' relies on elements that were already known in 1958.

As a matter of fact, the quite straightforward proof of the validity of the theoretical element that is called herein LHCD has apparently been unnoticed until the publication of [13]. This evidence looks like the reason for the rejection of the tensor interaction (11) as the basis for weak interactions (see e.g. [8], p. 219, [10]).

\section{Inherent Problems of the Electroweak Theory}

Two different weak interaction theories are shown above: the electroweak theory 
and the DDWIT. This section demonstrates the overwhelming advantage of the DDWIT over the electroweak theory. Arguments showing many cases where the electroweak theory is inconsistent with well established physical laws have already been published (see e.g. section 3 of [15] and references therein). Few examples of this inconsistency are briefly mentioned below. An argument that belongs to the Occam razor criterion is placed at the end.

1) The electroweak theory relies on the assumption of a massless neutrino. Few examples that are taken from the literature support this claim: A. Salam said in his Nobel lecture that the electroweak theory relies on "a neutrino which travels exactly with the velocity of light" [16]. A review article restates the neutrino masslessness attribute of the electroweak theory: "Two-component left-handed massless neutrino fields play crucial role in the determination of the charged current structure of the Standard Model" (see the Abstract of [17]). Similarly, a textbook states: "Neutrino masses are exactly zero in the Standard Model" (see [18], p. 533). Contrary to this assumption, it is now recognized that the neutrino is a massive particle [19].

2) The electroweak theory regards the $W^{ \pm}$bosons as electrically charged elementary particles. Unlike the case of the Dirac electron, the electroweak theory cannot prove that its equations of the $W^{\star}$ bosons conserve charge. It is well known that charge conservation is a fundamental element of Maxwellian electrodynamics. Hence, the electroweak theory violates Maxwellian electrodynamics.

3) The electroweak theory uses the $Z$ boson as an elementary particle whose quantum function takes a mathematically real form. The $Z$ boson is a massive particle, and its electroweak theory violates the de Broglie principle.

4) A coherent quantum theory is based on a differential equation whose solutions appropriately describe relevant physical processes. The Dirac theory of the electron is a good example of this issue. By contrast, no electroweak textbook shows an explicit form of the required differential equation. A fortiori, no solution to this unknown equation is tested with respect to relevant experimental data.

5) Consider the DDWIT Lagrangian density (12). It comprises just four terms, and its first three terms compose the QED Lagrangian density (6). The last term, which represents weak interactions, takes the same general form as that of the electromagnetic interaction term: Each of these terms is a Lorentz scalar which is a contraction of an external field with a tensor that depends on the Dirac $\gamma$ matrices.

The form of the electroweak Lagrangian density is completely different. It comprises more than 20 terms (see e.g. [20], p. 518; [21]). This unusual complexity is probably the reason that explains why quite a few Standard Model textbooks take the strange approach where they refrain from a presentation of the complete Lagrangian density of the electroweak theory.

The Occam razor principle says that in the case of two competing theories that have the same merit, one should prefer the simpler theory. This is a minor 
argument that is added to the above mentioned intrinsic errors of the electroweak theory.

\section{Conclusion}

Relativistic properties of the Lagrangian density of a quantum theory are compared with those of its Hamiltonian density. It is proved above that a Lorentz transformation of a Lagrangian density is not the same as that of a Hamiltonian density. This theoretical element is called herein the Lagrangian-Hamiltonian Covariance Difference (LHCD). This outcome has been unnoticed for a long time. It is also proved that LHCD affects the structure of weak interaction theories. In particular, the structure of the Pauli term of a Lagrangian (11) yields a Hamiltonian that is consistent with weak interaction data: The dimension of the interaction strength is $\left[L^{2}\right]$, which is the same as that of the Fermi coefficient $G_{F}$, and the Hamiltonian agrees with the V-A attribute of weak interactions. This theory takes the form of a Dipole-Dipole Weak Interaction Theory called DDWIT. The discussion of section 4 proves that the DDWIT is dramatically better than the Standard Model electroweak theory.

\section{Conflicts of Interest}

The authors declare no conflicts of interest regarding the publication of this paper.

\section{References}

[1] Wigner, E. (1960) The Unreasonable Effectiveness of Mathematics in the Natural Sciences. Communications on Pure and Applied Mathematics, 13, 1-14. https://doi.org/10.1002/cpa.3160130102

[2] Weinberg, S. (1995) The Quantum Theory of Fields. Vol. I, Cambridge University Press, Cambridge. https://doi.org/10.1017/CBO9781139644167

[3] Landau, L.D. and Lifshitz, E.M. (2005) The Classical Theory of Fields. Elsevier, Amsterdam.

[4] Bjorken, J.D. and Drell, S.D. (1965) Relativistic Quantum Fields. McGraw-Hill, New York. https://doi.org/10.1063/1.3047288

[5] Peskin, M.E. and Schroeder, D.V. (1995) An Introduction to Quantum Field Theory. Addison-Wesley, Reading.

[6] Bjorken, J.D. and Drell, S.D. (1964) Relativistic Quantum Mechanics. McGraw-Hill, New York.

[7] Thomson, M. (2013) Modern Particle Physics. Cambridge University Press, Cambridge. https://doi.org/10.1017/CBO9781139525367

[8] Perkins, D.H. (1987) Introduction to High Energy Physics. Addison-Wesley, Menlo Park.

[9] Wong, S.S.M. (1998) Introductory Nuclear Physics. Wiley, New York. https://doi.org/10.1002/9783527617906

[10] Feynman, R.P. and Gell-Mann, M. (1958) Theory of the Fermi Interaction. Physical Review, 109, 193-198. https://doi.org/10.1103/PhysRev.109.193 
[11] Wentzel, G. (1949) Quantum Theory of Fields. Interscience, New York.

[12] Pauli, W. (1941) Relativistic Field Theories of Elementary Particles. Reviews of Modern Physics, 13, 203-232. https://doi.org/10.1103/RevModPhys.13.203

[13] Comay, E. (2016) A Theory of Weak Interaction Dynamics. Open Access Library Journal, 3, 1-10. https://doi.org/10.4236/oalib.1103264 https://www.scirp.org/journal/PaperInformation.aspx?paperID=72788

[14] Comay, E. (2017) Further Aspects of Weak Interaction Dynamics. Open Access Library Journal, 4, 1-11. https://doi.org/10.4236/oalib.1103397 https://www.scirp.org/journal/PaperInformation.aspx?PaperID=74373

[15] Comay, E. (2019) Differences between Two Weak Interaction Theories. Physical Science International Journal, 21, 1-9. https://doi.org/10.9734/psij/2019/v21i130091 http://www.journalpsij.com/index.php/PSIJ/article/view/30091/56456

[16] Salam, A. (1979) Nobel Lecture. https://www.nobelprize.org/uploads/2018/06/salam-lecture.pdf

[17] Bilenky, S.M. (2015) Neutrino in Standard Model and Beyond. Physics of Particles and Nuclei, 46, 475-496. https://doi.org/10.1134/S1063779615040024

[18] Srednicki, M. (2007) Quantum Field Theory. Cambridge University Press, Cambridge. https://doi.org/10.1017/CBO9780511813917

[19] Formaggio, J.A. and Zeller, G.P. (2012) From eV to EeV: Neutrino Cross Sections across Energy Scales. Reviews of Modern Physics, 84, 1307-1341. https://doi.org/10.1103/RevModPhys.84.1307

[20] Sterman, G. (1993) An Introduction to Quantum Field Theory. Cambridge University Press, Cambridge. https://doi.org/10.1017/CBO9780511622618

[21] Wikipedia. The Electroweak Item. https://en.wikipedia.org/wiki/Electroweak_interaction\#After_electroweak_symmetr y_breaking14 